\title{
BMJ Open Determining a safe upper limit of oxygen supplementation for adult patients: a systematic review
}

\author{
Mathilde Languille Lassen (D) , ${ }^{1}$ Bjarke Risgaard, ${ }^{1}$ Josefine S Baekgaard, ${ }^{1}$ \\ Lars S Rasmussen ${ }^{1,2}$
}

To cite: Lassen ML,

Risgaard B, Baekgaard JS, et al. Determining a safe upper limit of oxygen supplementation for adult patients: a systematic review. BMJ Open 2021;11:e045057. doi:10.1136/ bmjopen-2020-045057

- Prepublication history for this paper is available online. To view these files, please visit the journal online (http://dx.doi. org/10.1136/bmjopen-2020045057).

Received 23 September 2020 Accepted 12 July 2021

Check for updates

(C) Author(s) (or their employer(s)) 2021. Re-use permitted under CC BY-NC. No commercial re-use. See rights and permissions. Published by BMJ.

${ }^{1}$ Department of Anaesthesia, Centre of Head and Orthopaedics, Rigshospitalet, Copenhagen, Denmark ${ }^{2}$ Department of Clinical Medicine, University of Copenhagen, Copenhagen, Denmark

Correspondence to Mathilde Languille Lassen; languillelassen@gmail.com

\section{ABSTRACT}

Objective This systematic review aimed to describe the connection between the inspired oxygen fraction and pulmonary complications in adult patients, with the objective of determining a safe upper limit of oxygen supplementation.

Methods MEDLINE and Embase were systematically searched in August 2019 (updated July 2020) for studies fulfilling the following criteria: intubated adult patients (Population); high fractions of oxygen (Intervention) versus low fractions of (Comparison); atelectasis, acute respiratory distress syndrome (ARDS), pneumonia and/ or duration of mechanical ventilation (utcome); original studies both observational and interventional (Studies). Screening, data extraction and risk of bias assessment was done by two independent reviewers.

Results Out of 6120 records assessed for eligibility, 12 were included. Seven studies were conducted in the emergency setting, and five studies included patients undergoing elective surgery. Eight studies reported data on atelectasis, two on ARDS, four on pneumonia and two on duration of mechanical ventilation. There was a non-significant increased risk of atelectasis if an oxygen fraction of 0.8 or above was used, relative risk (RR): 1.37 (95\% Cl 0.95 to 1.96). One study showed an almost threefold higher risk of pneumonia in the high oxygen fraction group (RR: $2.83(95 \% \mathrm{Cl} 2.25$ to 3.56$)$ ). The two studies reporting ARDS and the two studies with data on mechanical ventilation showed no association with oxygen fraction. Four studies had a high risk of bias in one domain. Conclusions In this systematic review, we found inadequate evidence to identify a safe upper dosage of oxygen, but the identified studies suggest a benefit of keeping inspiratory oxygen fraction below 0.8 with regard to formation of atelectases.

PROSPERO registration number CRD42020154242.

\section{INTRODUCTION}

Oxygen is a molecule vital for life, as it is the cornerstone in cellular respiration in all aerobic organisms. In trauma care, during anaesthesia and in the management of respiratory failure, an oxygen fraction $\left(\mathrm{FiO}_{2}\right)$ of 0.21 may not be sufficient to maintain an acceptable oxygen concentration in arterial blood and oxygen supplementation is therefore often part of standard care. ${ }^{12}$

\section{Strengths and limitations of this study}

- The use of predefined Population, Intervention, Comparison, Outcome and Study design to asses studies for eligibility.

- The use of a wide search string in two databases.

- Two independent reviewers screening and including studies, assessing risk of bias and extracting data.

- There is a risk of publication bias that arises due to the possibility of missing unpublished studies.

- It is possible that our search did not identify all relevant studies.

Supplementary oxygen may result in hyperoxaemia, with the risk of tissue hyperoxia. An increasing amount of evidence has connected hyperoxia and hyperoxaemia with increased mortality $^{3-6}$ possibly as a consequence of a variety of factors associated with hyperoxia: atelectasis in the lungs, ${ }^{78}$ formation of reactive oxygen species, ${ }^{9}$ impairment of the innate immune system, ${ }^{10}$ as well as vasoconstriction with paradox tissue hypoxia to follow. ${ }^{11}$

All in all, hypoxia should be avoided, but at the same time it seems that exposure to high concentrations of oxygen may have serious consequences. Therefore, it is relevant to investigate if a safe upper dosage of oxygen can be identified.

This systematic review aimed to describe the connection between the $\mathrm{FiO}_{2}$ and pulmonary complications in intubated adult patients, with the objective of determining a safe upper limit of oxygen supplementation. We defined pulmonary complications as atelectasis, pneumonia and acute respiratory distress syndrome (ARDS).

\section{METHODS}

Protocol and registration

Methods of the analysis and inclusion criteria were prespecified and documented in a protocol. The protocol was completed 
following the Preferred Reporting Items for Systematic Reviews and Meta-Analysis guidelines for protocols. ${ }^{12} \mathrm{a}^{13}$

\section{Eligibility criteria}

Studies were selected according to following predefined Population, Intervention, Comparison, Outcome and Study design (PICOS).

\section{Inclusion criteria}

- Population: intubated patients $\geq 18$ years.

- Intervention and Comparison: low inspiratory $\mathrm{FiO}_{2}$ (as defined by author) versus high $\mathrm{FiO}_{2}$ (as defined by authors).

- Outcome: atelectasis, pneumonia, ARDS and duration of mechanical ventilation (as defined by authors).

- Study design: original studies both interventional and observational.

\section{Exclusion criteria}

- Hyperbaric oxygen treatment.

- Case reports, review articles and editorials.

We had no restrictions on year of publication. The search was restricted to studies published in French, English or Danish.

\section{Information sources and search}

We searched MEDLINE and Embase using the following predefined search string (presented search strategy is from MEDLINE).

1. $(((((($ oxygen [Title/Abstract]) OR oxygen[MeSH Terms]) OR hyperoxia[Title/Abstract]) OR "supplemental oxygen"[Title/Abstract]) OR "oxygen supplementation"[Title/Abstract]) OR fio2[Title/ Abstract $])))$

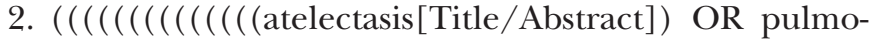
nary atelectasis[MeSH Terms]) OR pneumonia[Title/ Abstract]) OR pneumonia[MeSH Terms]) OR "lung collapse"[Title/Abstract]) OR "collapsed lung"[Title/ Abstract]) OR "acute lung injury" [Title/Abstract]) OR acute lung injury[MeSH Terms]) OR ARDS[Title/Abstract]) OR "acute respiratory distress syndrome" [Title/Abstract]) OR respiratory distress syndrome, adult[MeSH Terms])))

3. (intub*) OR "mechanical ventilation"

4. \#1 AND \#2 AND \#3.

The search was done the 6 August 2019. The search was updated the 6 July 2020. Modifications were made to fit Embase.

We identified one additional record ${ }^{14}$ by obtaining the full-text article of an abstract identified through the search string. Another record ${ }^{15}$ was identified by screening the reference list of an article.

\section{Selection process}

Two independent reviewers (MLL and BR) screened all titles and abstracts yielded by the search against the inclusion criteria using Covidence (an online programme facilitating the production of systematic reviews developed by the Cochrane group). ${ }^{16}$ A Cohen's Kappa for inter-rater reliability was calculated. The same reviewers obtained full text articles for all titles that appeared to meet the inclusion criteria or where there was any uncertainty. Disagreements were resolved through discussion until consensus. All full-text articles were assessed by the same two independent reviewers and those not meeting the inclusion criteria were excluded.

\section{Data collection and data items}

Data extraction was done by two authors (MLL and BR), and was facilitated by the data-extraction tool Covidence and by using predefined forms. We collected study characteristics including trial design, trial size, country, period and year of publication. From the included studies we extracted the dosage of oxygen, type of control used, duration of treatment, patient characteristics (gender, age, patient type) as well as data on the predefined outcomes (atelectasis, pneumonia, ARDS) as defined by the authors.

\section{Risk of bias}

Risk of bias for non-randomised studies were assessed by using the Newcastle-Ottawa Scale. ${ }^{17}$ Here each study can be awarded from zero to nine stars, with zero stars representing a high risk of bias, and nine stars a low risk. Each study can be judged and awarded stars on eight items, categorised into three domains: selection of the study group, comparability of cohorts, and evaluation of the outcome of interest.

For randomised studies we used the Cochrane Collaboration tool for assessing risk of bias (Table 8.5.a in the Cochrane Handbook for Systematic Reviews of Intervention) in Covidence, which covers: sequence generation, allocation concealment, blinding, incomplete data and selective outcome reporting. A judgement as to the possible risk of bias on each domain were made from the extracted information, rated as 'high risk', 'low risk' or 'unclear' risk of bias. These judgements were made based on the criteria for judging the risk of bias (Table 8.5.d in the Cochrane Handbook Higgins 2011).

\section{Summary measures and synthesis of results}

This systematic review was expected to be a descriptive summary of the current evidence on oxygen supplementation and pulmonary complications. Relative risk (RR) was calculated where possible and a forest plot was used to illustrate the results. RRs with $95 \%$ CIs, was calculated in studies where this information was missing and the calculation was possible. The forest plot was made with a random-effects model.

\section{Patient and public involvement}

No patient involved.

\section{RESULTS}

\section{Study selection}

Our initial search strategy identified 7734 records. After duplicates were removed and two additional records from 


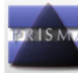

PRISMA 2009 Flow Diagram
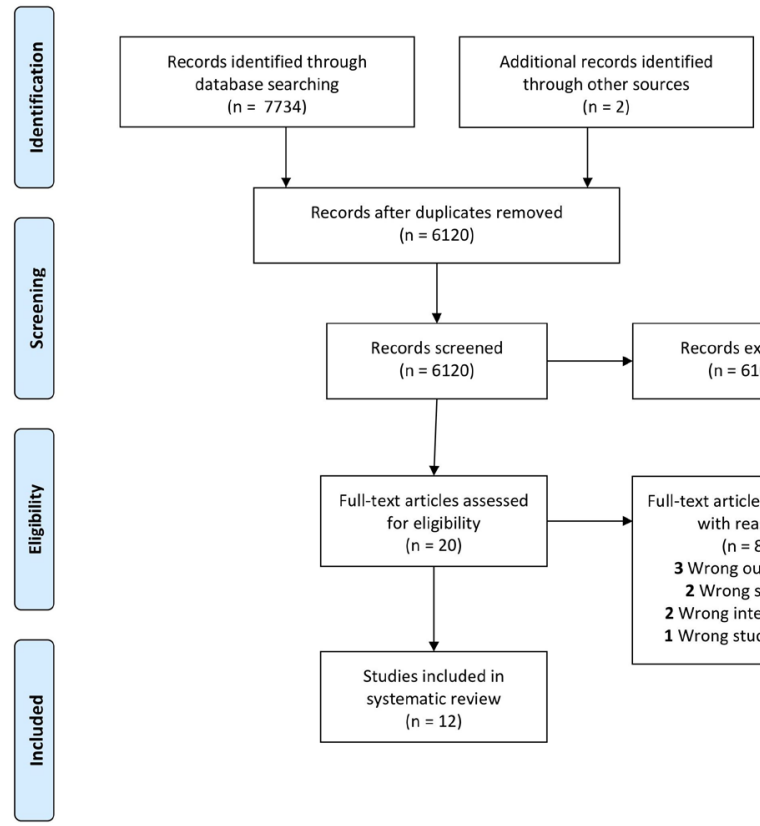

Figure 1 Preferred Reporting Items for Systematic Reviews and Meta-analyses (PRISMA) flow diagram of identification, screening, eligibility and inclusion process ${ }^{28}$ on a search for studies comparing low dose oxygen supplementation with high dose oxygen supplementation with pulmonary complications as an outcome. ${ }^{37}$

other sources were added, 6120 records were screened. Of these, 6100 were excluded as they did not fulfil eligibility criteria leaving 20 records for full-text screening. Cohen's kappa for inter-rater reliability of 0.43 (95\% CI 0.26 to 0.60 ) was calculated, which is judged to be moderate agreement. After full-text review, 12 records fulfilled the inclusion criteria (figure 1).

\section{Study characteristics}

Study characteristics are summarised in table 1. Eight of the 12 included studies were randomised controlled trials. Among the four remaining there were two retrospective observational studies ${ }^{18}$ and two prospective observational studies. ${ }^{20}{ }^{21}$ About half of the studies were conducted in Europe. Seven studies were conducted in the acute care setting. Of these seven, one study ${ }^{22}$ included patients with septic shock, four studies ${ }^{14} 182123$ recruited surgical, medical and trauma patients that were mechanically ventilated in the intensive care unit, one study ${ }^{20}$ included patients with acute lung injury and the last study ${ }^{24}$ recruited patients with traumatic brain injury. The remaining five studies included patients undergoing different types of elective surgery.

The administered $\mathrm{FiO}_{2}$ varied substantially among the studies, with $\mathrm{FiO}_{2}$ ranging from 0.26 to 0.60 in the low $\mathrm{FiO}_{2}$ group and from 0.36 to 1.0 in the high $\mathrm{FiO}_{2}$ group.

Table 2 presents the outcomes of interest reported in the included studies. Eight studies reported on the incidence of atelectasis, two studies reported on ARDS, four studies reported on pneumonia and two studies reported on the duration of mechanical ventilation.

\section{Atelectasis}

The eight studies reporting on atelectasis, generally showed better outcomes for patients in the low $\mathrm{FiO}_{2}$ group, as two studies ${ }^{22} 25$ showed almost two-fold higher risk of atelectasis in the high $\mathrm{FiO}_{2}$, with RR: 1.875 (95\% CI 0.42 to 8.37 ) and RR: 2.0 (95\% CI 1.06 to 3.79), respectively. One study ${ }^{15}$ suggested a minor benefit of treatment with low $\mathrm{FiO}_{2}$, but this was not statistically significant, RR: 1.46 (95\% CI 0.97 to 2.20$)$. Another study ${ }^{24}$ found RR: 0.91 (95\% CI 0.56 to 1.50 ) suggesting a benefit of treatment with high $\mathrm{FiO}_{2}$, but this was not statistically significant. These studies are illustrated in the forest plot (figure 2), which shows that in general treatment with high $\mathrm{FiO}_{2}$ was associated with higher risk of atelectasis formation, RR:1.37 (95\% CI 0.95 to 1.96). The heterogeneity (I2) of the meta-analysis presented in figure 2 is $31 \%$, which corresponds to a moderate heterogeneity (Cochrane Handbook for Systematic Reviews of Intervention, section 9.5.2 Identifying and measuring heterogeneity).

Rothen $e t a l^{8}$ found a 16.8 times greater area of atelectasis in the high $\mathrm{FiO}_{2}$ group and similarly, the study by Benoît $e t a l^{26}$ found a threefold larger atelectatic surface in the high $\mathrm{FiO}_{2}$ group. Suzuki et $a l^{21}$ estimated atelectasis as time-weighted averages, and also found a beneficial effect of a low $\mathrm{FiO}_{2}$. In the study by Ishii et $a l^{18}$ additional information on intubated patients were found in an abstract $^{27}$ from the same study. They found a higher incidence of atelectasis in the high $\mathrm{FiO}_{2}$ group, but the total number of patients was not reported.

\section{Acute respiratory distress syndrome}

Panwar et $a l^{14}$ showed an increase of new-onset ARDS in the low $\mathrm{FiO}_{2}$ group, RR: 0.87 (95\% CI 0.43 to 1.75 ), but this was not statistically significant. The study by Lång et $a l^{24}$ found three patients with ARDS in the low $\mathrm{FiO}_{2}$ group, while no patients with ARDS were identified in the group receiving high $\mathrm{FiO}_{2}$.

\section{Pneumonia}

The study by Staehr-Rye et $a l^{19}$ showed a significant increase in the incidence of pneumonia, RR: $2.83(95 \%$ CI 2.25 to 3.56) in the high $\mathrm{FiO}_{2}$ group. Similarly, Barrot et $a l^{23}$ showed a small, but non-significant, tendency to ventilator-associated pneumonias in the high $\mathrm{FiO}_{2}$ group, RR: 1.26 (95\% CI 0.71 to 2.22). The two other studies, Asfar $e t a l^{22}$ and Lång et $a l l^{24}$ found a non-significant tendency for pneumonia in the low $\mathrm{FiO}_{2}$ group with RR: 0.94 (95\% CI 0.59 to 1.49 ) and RR: 0.71 (95\% CI 0.26 to 1.97$)$, respectively. These studies are illustrated in the forest plot (figure 3), which shows a non-significant tendency that treatment with high $\mathrm{FiO}_{2}$ was associated with higher risk of pneumonia, RR: 1.32 (95\% CI 0.65 to 2.70). 


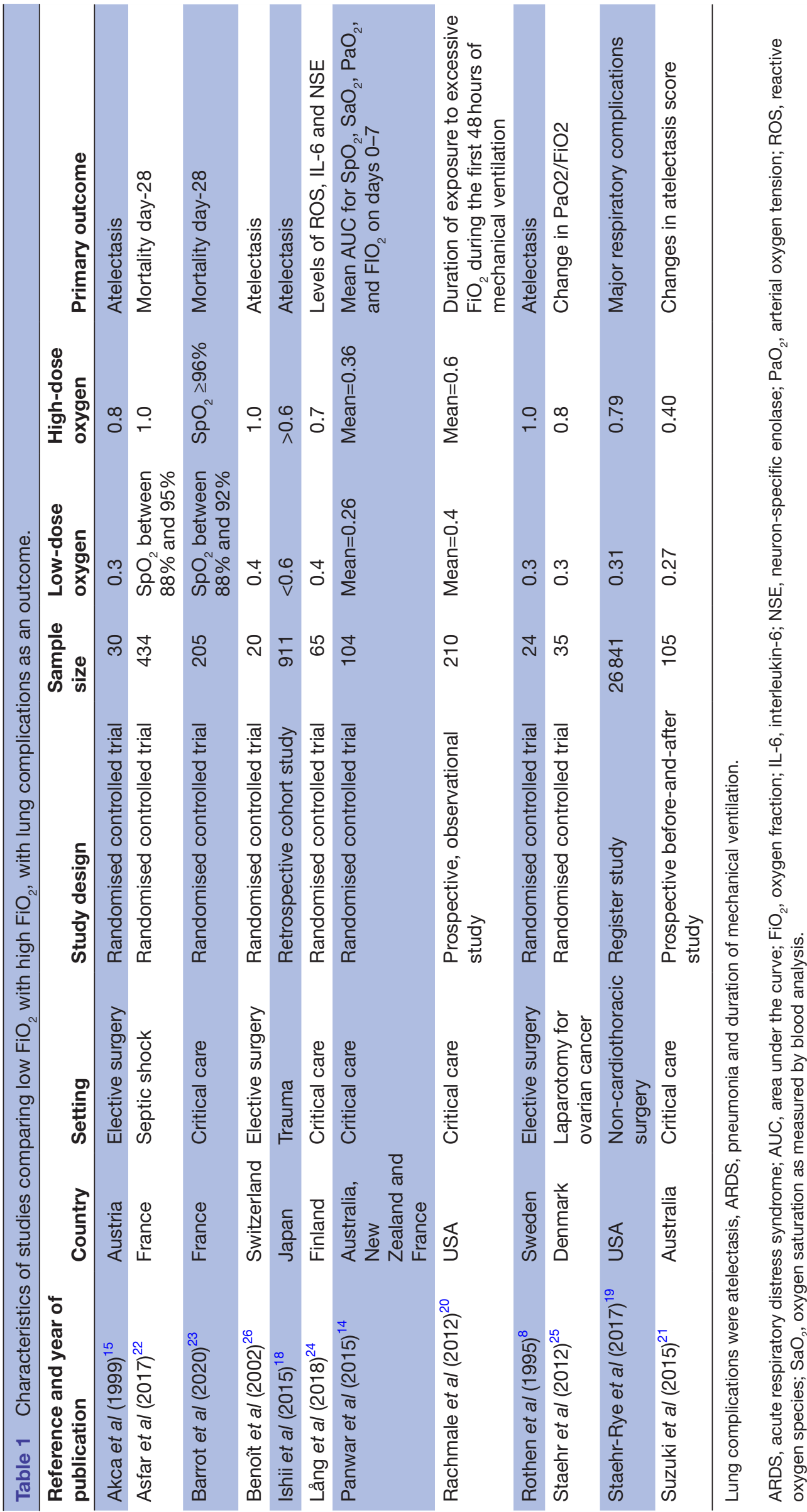


Table 2 Patient outcomes comparing low doses of oxygen supplementation with high doses of oxygen supplementation

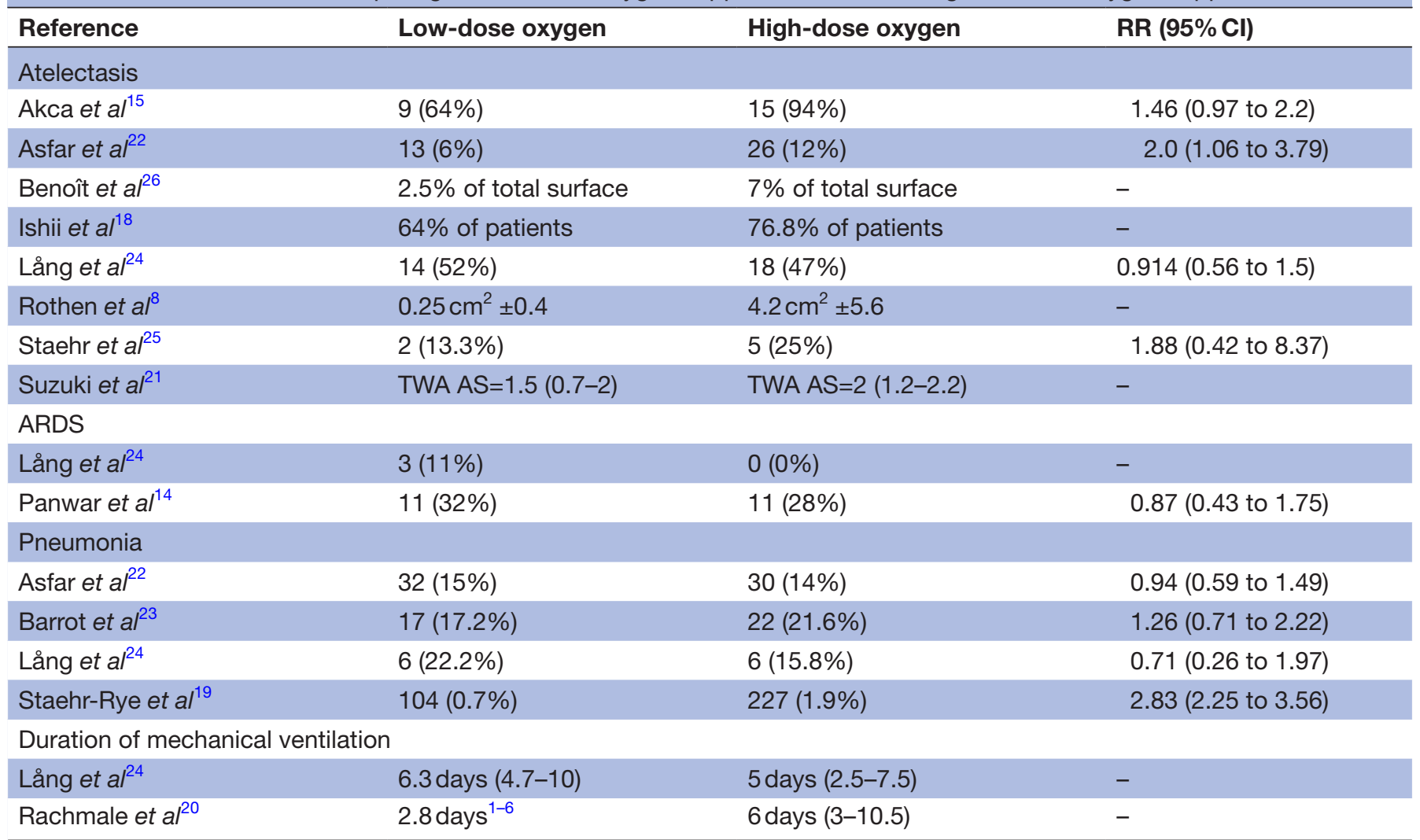

Continuous data are presented as mean (SD) or median (IQR). RR is presented with high-dose oxygen in the numerator.

ARDS, acute respiratory distress syndrome; RR, relative risk; TWA AS, time-weighted average atelectasis.

\section{Duration of mechanical ventilation}

The two studies reporting the duration of mechanical ventilation pointed in opposite direction. Lång et $a l^{24}$ reported slightly more time spent on mechanical ventilation in the low $\mathrm{FiO}_{2}$ group, while Rachmale $e t a l^{20}$ reported a twofold increase in time in the high $\mathrm{FiO}_{2}$ group.

\section{Risk of bias assessment}

Risk of bias for randomised studies are illustrated in table 3. Three studies had no blinding of participants, personal or outcome assessment, leaving them with a high risk of bias on these domains. ${ }^{81422}$ In the study by Rothen $e t a l^{8}$ it was unclear if a randomisation was performed between the low $\mathrm{FiO}_{2}$ group and the high $\mathrm{FiO}_{2}$ group, indicating a high risk of bias.

Lång $e t a l^{24}$ was an open-label trial, and was therefore awarded a high risk of bias on the domain of blinding of participants and personnel, however, the outcome assessor was blinded.

The four non-randomised studies were assessed using the New-Castle Ottawa Scale. ${ }^{17}$ One study ${ }^{20}$ scored six stars, two studies ${ }^{18} 19$ scored seven stars and one study ${ }^{21}$ scored eight stars, indicating an overall high quality of the studies.

\section{DISCUSSION}

\section{Summary of findings}

In this study, we were not able to determine a safe upper limit of oxygen supplementation, due to inadequate evidence and heterogeneity as the included studies had different endpoints with varying definitions, and also different ways of defining low and high $\mathrm{FiO}_{2}$. In some

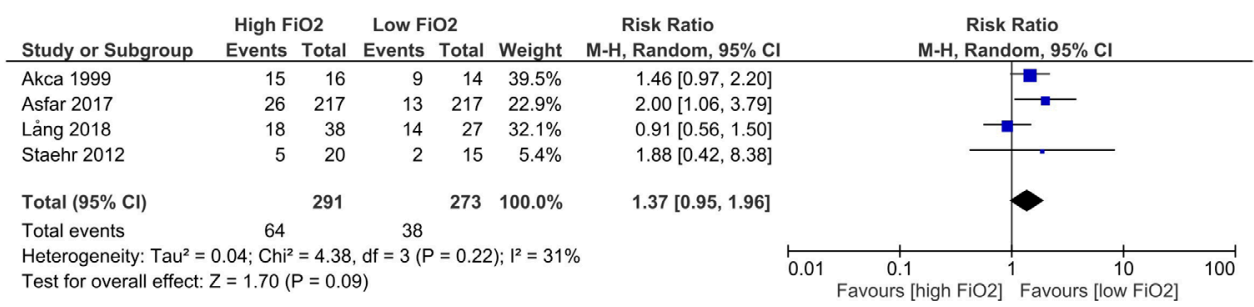

Figure 2 Forest plot of formation of atelectasis in studies comparing low $\mathrm{FiO}_{2}$ with high $\mathrm{FiO}_{2}$. $\mathrm{FiO}_{2}$, oxygen fraction; $\mathrm{M}$. $\mathrm{H}$, Maentel-Haentzel. 


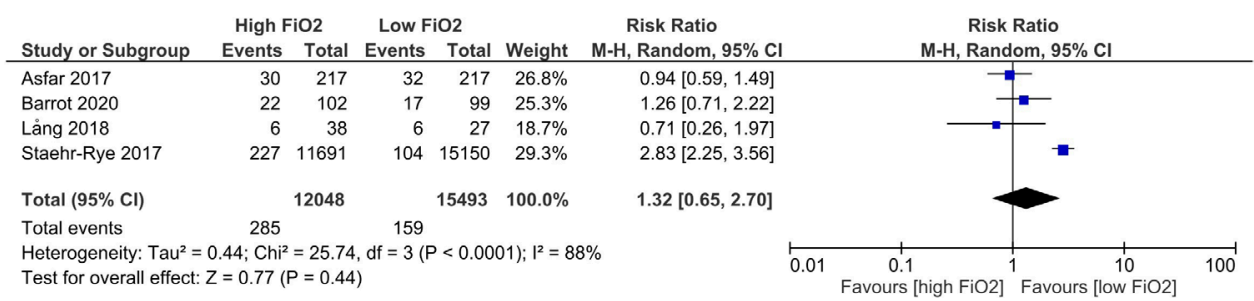

Figure 3 Forest plot of risk of pneumonia in studies comparing low $\mathrm{FiO}_{2}$ with high $\mathrm{FiO}_{2}$. $\mathrm{FiO}_{2}$, oxygen fraction.; M.H. Random, Maentel-Haentzel.

studies the $\mathrm{FiO}_{2}$ in the low $\mathrm{FiO}_{2}$ group was higher than in the high $\mathrm{FiO}_{2}$ group in other studies.

Regarding atelectasis, seven of the eight studies favoured a conservative oxygen strategy with low $\mathrm{FiO}_{2}$ and an $\mathrm{FiO}_{2}$ above 0.8 seemed to be associated with higher risk of atelectasis formation. Looking at figure 2, there is an RR of 1.37 , which suggests a clinically relevant difference with less atelectasis with a lower $\mathrm{FiO}_{2}$. However, the $\mathrm{CI}$ is wide (0.95-1.96), indicating that more information is needed before any firm conclusions can be made.

\section{Strengths and limitations}

This study was conducted according to the Preferred Reporting Items for Systematic Reviews and Meta-analyses guidelines ${ }^{28}$ ensuring a systematic and broadly acknowledged approach to the present literature. The strengths of this approach include predefined PICOS criteria to assess study eligibility, use of a wide search string in two databases and two independent reviewers screened and assessed studies, including risk of bias.

Our study is limited by general weaknesses of systematic reviews. This includes risk of publication bias that arises due to the possibility of missing non-published studies. Despite the systematic search with predefined search string, and screening of reference lists of included studies, there is always a possibility that our search did not identify all relevant studies. However, the heterogeneity of the 12 studies reviewed makes us believe that potentially missed studies would not change the conclusion substantially. It is possible that more studies could have been found by searching in a wider set of databases. However, we chose the most commonly used databases MEDLINE and EMBASE, where the quality is known to be best and where most studies are found.

The patient population was determined in very broad terms (intubated adult patients), resulting in more heterogeneity among the included studies.

The trials varied in patient groups, associated clinical care and disease severity. Furthermore, in some studies it is unclear when exactly the outcome of interest was measured (early or late onset of ARDS and timing of CT/X-ray for measuring the presence of atelectasis). It is also unclear how pneumonia was defined in the four

Table 3 Risk of bias assessment for randomised controlled trials comparing low dose oxygen supplementation with high dose oxygen supplementation

Random sequence generation
Bllocation concealment
Blinding of outcome assessment
Selective reporting
Other bias

Risk of bias was assessed using Cochrane Collaboration tool for assessing risk of bias (Table 8.5.a in the Cochrane Handbook for Systematic Reviews of Intervention).

Green, low risk of bias; Red, high risk of bias; Yellow, unclear risk of bias. 
studies reporting this outcome. Therefore, conclusions should be drawn with caution.

Half of the randomised controlled trials were not blinded to personnel and participants, increasing the risk of performance bias. Three of these were not blinded to outcome assessors which increase the risk of detection bias. In general, many of the studies are relatively small, increasing the risk of other bias such as publication bias (table 3).

Atelectasis was defined in different ways complicating the pooling of data and the possibility to undertake a meta-analysis. Three studies ${ }^{8} 1525$ used CT-scans and they all considered densities between -100 and +100 Hounsfield as atelectasis. Of these three, one ${ }^{8}$ measured areas of atelectasis in $\mathrm{cm}^{2}$ whereas the two others ${ }^{15} 25$ measured if atelectases were present or not. Ishii et $a l^{18}$ also used CT-scans, but defined atelectases as areas with formation of more than $10 \mathrm{~mm}$ thick atelectasis from the first to the second scan. The study by Staehr $e t a l^{25}$ did not define specific criteria on when densities were judged as atelectasis or not.

Asfar $e t a l^{22}$ and Suzuki $e t a l^{21}$ used chest X-rays, without defining atelectasis specifically, as this was decided by the individual physician. Lång $e t a l^{24}$ used chest X-rays in the same manner, however they allowed the appliance of positive end-expiratory pressure to minimise atelectasis, which makes it hard to directly compare results with other studies. Only Suzuki et $a l^{21}$ used more than one radiologist to perform the outcome assessment.

In Panwar et al, ${ }^{14}$ new-onset ARDS was defined as subsequent occurrence of ARDS in those patients who did not have ARDS on day 0, and where ARDS was present according to the Berlin definition. ${ }^{29}$ Lång $e t a l^{4}$ did not report their definition of ARDS.

Regarding pneumonia, the database study of 26841 patients performed by Staehr-Rye $e t a l^{19}$ found a significant, almost threefold higher risk of pneumonia in the liberal oxygen group, indicating that excess levels of oxygen may be harmful. However, this is an analysis of administrative data, with risk of misclassification bias, and therefore, direct conclusions should be drawn with caution.

\section{Other reviews}

The evidence for the use of supplemental oxygen has been investigated in recently published systematic reviews. A systematic review and meta-analysis by Damiani et $a l^{30}$ from 2014 suggests an association between hyperoxia and mortality in patients with stroke, traumatic brain injury and those resuscitated from cardiac arrest. However, they concluded that their results were limited by the heterogeneity of the included studies. The same conclusion was drawn in another meta-analysis from 2015 by Helmerhorst $e t$ al. ${ }^{31}$ No definite conclusions could be made due to heterogeneity in the included studies; however, the meta-analysis suggested a benefit of conservative oxygen therapy. In a Cochrane review from 2015 by Wetterslev et $a l^{32}$ comparing low $\left(\mathrm{FiO}_{2} 0.30-0.40\right)$ vs high $\left(\mathrm{FiO}_{2}\right.$
0.60-0.90) perioperative inspiratory $\mathrm{FiO}_{2}$, they found no association between perioperative $\mathrm{FiO}_{2}$ and postoperative surgical site infection and mortality. In another Cochrane review from 2016 performed by Cabello et $a l^{33}$, they focused on patients with acute myocardial infarctions. They included five studies and found no clear recommendations on the use of oxygen supplementation.

In a recent meta-analysis performed in 2018 by $\mathrm{Chu}$ et $a l,{ }^{4}$ they included 25 randomised controlled trials on acutely ill patients and found a significant association between liberal oxygenation strategies and increased mortality in-hospital, at 30 days and at longest follow-up. Nevertheless, morbidity outcomes were similar between groups.

The available reviews are limited because of heterogeneity, including different outcome measures, overall indicate that excess oxygen is harmful, stressing the need for further investigation on this subject.

Oxygen supplementation is obviously a vital part of trauma care, practice of anaesthesia, the management of respiratory distress and treatment of a variety of other conditions. However, supplemental oxygen should be carefully considered a drug and prescribed adequately. There is a general lack of strong evidence for supplemental oxygen, and an upper limit of oxygen supplementation is not included in many guidelines. ${ }^{134-36}$ Our study contributes to the current evidence in a different way, by looking at the association between $\mathrm{FiO}_{2}$ and pulmonary complications, which is a highly relevant indicator in the search for a safe upper limit of oxygen supplementation.

As oxygen supplementation is so widely used, it is crucial that better evidence-based guidelines are developed. Future research is required to precisely define the oxygen therapy strategies to maximise benefits and minimise harms.

\section{Conclusion}

In this systematic review, we found that there was inadequate evidence to identify a safer upper dosage of oxygen, but the identified studies suggest a benefit of conservative oxygen therapy, defined as $\mathrm{FiO}_{2} \leq 0.8$ with regard to formation of atelectasis.

Contributors The idea for the article was formed by LSR. Literature search was performed by MLL and BR. MLL wrote the article. JSB contributed with high subject knowledge and all authors gave substantial contributions to the work. Every author revised the work critically for important intellectual content. Every author made a final approval of the version to be published. Every author agreed to be accountable for all aspects of the work.

Funding Departmental funding. Award/Grant number is not applicable.

Competing interests None declared.

Patient and public involvement Patients and/or the public were not involved in the design, or conduct, or reporting, or dissemination plans of this research.

Patient consent for publication Not required.

Provenance and peer review Not commissioned; externally peer reviewed.

Data availability statement Data are available on reasonable request. This was a systematic review and researchers can contact the authors to access the material.

Open access This is an open access article distributed in accordance with the Creative Commons Attribution Non Commercial (CC BY-NC 4.0) license, which 
permits others to distribute, remix, adapt, build upon this work non-commercially, and license their derivative works on different terms, provided the original work is properly cited, appropriate credit is given, any changes made indicated, and the use is non-commercial. See: http://creativecommons.org/licenses/by-nc/4.0/.

\section{ORCID iD}

Mathilde Languille Lassen http://orcid.org/0000-0001-6004-8856

\section{REFERENCES}

1 O'Driscoll BR, Howard LS, Earis J. British thoracic society guideline for oxygen use in adults in healthcare and emergency settings. BMJ Open Respir Res 2017;4:1-20.

2 Thim T, Krarup NHV, Grove EL, et al. Initial assessment and treatment with the airway, breathing, circulation, disability, exposure (ABCDE) approach. Int J Gen Med 2012;5:117-21.

3 Brenner M, Stein D, Hu P, et al. Association between early hyperoxia and worse outcomes after traumatic brain injury. Arch Surg 2012;147:1042-6.

4 Chu DK, Kim LH-Y, Young PJ, et al. Mortality and morbidity in acutely ill adults treated with liberal versus conservative oxygen therapy (IOTA): a systematic review and meta-analysis. Lancet 2018;391:1693-705

5 Wang $\mathrm{C}-\mathrm{H}$, Chang $\mathrm{W}-\mathrm{T}$, Huang $\mathrm{C}-\mathrm{H}$, et al. The effect of hyperoxia on survival following adult cardiac arrest: a systematic review and metaanalysis of observational studies. Resuscitation 2014;85:1142-8.

6 Girardis M, Busani S, Damiani E, et al. Effect of conservative vs conventional oxygen therapy on mortality among patients in an intensive care unit: the oxygen-ICU randomized clinical trial. JAMA 2016;316:1583-9.

7 Aboab J, Jonson B, Kouatchet A, et al. Effect of inspired oxygen fraction on alveolar derecruitment in acute respiratory distress syndrome. Intensive Care Med 2006;32:1979-86.

8 Rothen HU, Sporre B, Engberg G, et al. Prevention of atelectasis during general anaesthesia. Lancet 1995;345:1387-91.

9 Turrens JF. Mitochondrial formation of reactive oxygen species. J Physiol 2003;552:335-44.

10 Baleeiro CEO, Wilcoxen SE, Morris SB, et al. Sublethal hyperoxia impairs pulmonary innate immunity. J Immunol 2003;171:955-63.

11 Ariyaratnam P, Loubani M, Bennett R. Hyperoxic vasoconstriction of human pulmonary arteries: a novel insight into acute ventricular septal defects. ISRN Cardiol 2013;2013:1-4

12 Shamseer L, Moher D, Clarke M, et al. Preferred reporting items for systematic review and meta-analysis protocols (PRISMA-P) 2015: elaboration and explanation. BMJ 2015;349:g7647.

13 PROSPERO. International prospective register of systematic reviews. Available: https://www.crd.york.ac.uk/prospero/

14 Panwar R, Hardie M, Bellomo R, et al. Conservative versus libera oxygenation targets for mechanically ventilated patients. A pilot multicenter randomized controlled trial. Am J Respir Crit Care Med 2016;193:43-51.

15 Akça O, Podolsky A, Eisenhuber E, et al. Comparable postoperative pulmonary atelectasis in patients given $30 \%$ or $80 \%$ oxygen during and 2 hours after colon resection. Anesthesiology 1999;91:991-8.

16 Covidence systematic review software. Veritas health innovation. Melbourne. Available: www.covidence.org

17 Wells GA, Shea B, O'Conell D. The Newcastle-Ottawa scale (NOS) for assessing the quality if nonrandomized studies in meta-analyses. Evidence-based public health, 2012. Available: http://www.evidence basedpublichealth.de/download/Newcastle_Ottowa_Scale_Pope_ Bruce.pdf
18 Ishii K, Morimatsu H, Ono K, et al. Relationship between a highinspired oxygen concentration and dorsal atelectasis in high-energy trauma patients. Acta Med Okayama 2020;74:17-26.

19 Staehr-Rye AK, Meyhoff CS, Scheffenbichler FT, et al. High intraoperative inspiratory oxygen fraction and risk of major respiratory complications. Br J Anaesth 2017;119:140-9.

20 Rachmale S, Li G, Wilson G. Practice of excessive FIO2 and effect on pulmonary outcomes in mechanically ventilated patients with acute lung injury. Respir Care 2012;57:1887-93.

21 Suzuki S, Eastwood GM, Goodwin MD, et al. Atelectasis and mechanical ventilation mode during conservative oxygen therapy: a before-and-after study. J Crit Care 2015;30:1232-7.

22 Asfar P, Schortgen F, Boisramé-Helms J, et al. Hyperoxia and hypertonic saline in patients with septic shock (HYPERS2S): a twoby-two factorial, multicentre, randomised, clinical trial. Lancet Respir Med 2017;5:180-90.

23 Barrot L, Asfar P, Mauny F, et al. Liberal or conservative oxygen therapy for acute respiratory distress syndrome. $N$ Engl J Med 2020;382:999-1008.

24 Lång M, Skrifvars MB, Siironen J, et al. A pilot study of hyperoxemia on neurological injury, inflammation and oxidative stress. Acta Anaesthesiol Scand 2018;62:801-10.

25 Staehr AK, Meyhoff CS, Henneberg SW, et al. Influence of perioperative oxygen fraction on pulmonary function after abdominal surgery: a randomized controlled trial. BMC Res Notes 2012;5:383.

26 Benoît Z, Wicky S, Fischer J-F, et al. The effect of increased Fio2 before tracheal extubation on postoperative atelectasis. Anesthesia \& Analgesia 2002;95:1777-81.

27 Ishii K, Morimatsu H, Ono K. Relationship between a high inspired oxygen concentration and a gravity dependent atelectasis in trauma patients: a subgroup analysis. 2015 Annu Meet Int Anesth Res Soc IARS 2015 Honolulu, HI United States 2015;120:S109.

28 Moher D, Liberati A, Tetzlaff J. Preferred reporting items for systematic reviews and meta-analyses: the PRISMA statement. Int J Surg 2010;8:658

29 Rawal G, Yadav S, Kumar R. Acute respiratory distress syndrome: an update and review. J Trans/ Intern Med 2018;6:74-7.

30 Damiani E, Adrario E, Girardis M, et al. Arterial hyperoxia and mortality in critically ill patients: a systematic review and metaanalysis. Crit Care 2014;18.

31 Helmerhorst HJF, Roos-Blom MJ, Van Westerloo DJ. Association between arterial hyperoxia and outcome in subsets of critical illness: a systematic review, meta-analysis, and meta-regression of cohort studies. Crit Care Med 2015;43:1508-19.

32 Wetterslev J, Meyhoff CS, Jørgensen LN, et al. The effects of high perioperative inspiratory oxygen fraction for adult surgical patients. Cochrane Database Syst Rev 2015;6:CD008884.

33 Cabello JB, Burls A, Emparanza Jl, et al. Oxygen therapy for acute myocardial infarction. Cochrane Database Syst Rev 2016;12:CD007160.

34 Casaubon LK, Boulanger J-M, Blacquiere D, et al. Canadian stroke best practice recommendations : Hyperacute Stroke Care Guidelines, Update 2015. Int J Stroke 2015;10:924-40.

35 Nikolaou NI, Welsford M, Beygui F, et al. Part 5: acute coronary syndromes: 2015 international consensus on cardiopulmonary resuscitation and emergency cardiovascular care science with treatment recommendations. Resuscitation 2015;95:e121-46.

36 Moga C, Chojecki D. Oxygen therapy in acute care settings. Institute of health economics, 2016. Available: https://www.ihe.ca/ publications/oxygen-therapy-in-acute-care-settings

37 Moher D, Liberati A, Tetzlaff J, et al. Preferred reporting items for systematic reviews and meta-analyses: the PRISMA statement. PLoS Med 2009;6:e1000097. 